\title{
ORIGINAL
}

\section{EFECTO DEL CONSUMO DE UNA HARINA DE MAÍZ ENRIQUECIDA CON SOJA EN EL ESTADO DE NUTRICIÓN DE MUJERES INDÍGENAS DE MÉXICO}

\begin{abstract}
María del Refugio Carrasco Quintero (1), Luis Ortiz Hernández (2), José Antonio Roldán Amaro (1), Adolfo Chávez Villasana (1), Judith Aguirre Arenas (1) y Francisco Raúl Aguilar Carrasco (1).
\end{abstract}

(1) Instituto Nacional de Ciencias Médicas y Nutrición. México Distrito Federal.

(2) Universidad Autónoma Metropolitana. México Distrito Federal.

No existen conflictos de interés.

Este trabajo fue financiado por DICONSA, antes CONASUPO (Compañía Nacional de Subsidios Populares).C/COL/2907/2010.

RESUMEN
Fundamentos: El problema de la deficiencia de micronutrientes en la
dieta habitual tiene un alto impacto, en México afecta a más de 28 millones de
habitantes, principalmente mujeres indígenas y de zonas rurales. Una de las
soluciones es la suplementación alimentaria. El objetivo del estudio fue eva-
luar el impacto de una harina de maíz enriquecida con proteína de soja en mu-
jeres indígenas de áreas rurales.

Método: El estudio se llevó a cabo en el 2010 con una muestra de 308 mujeres mexicanas que vivían en el medio rural, fue de intervención, longitudinal, doble ciego y aleatorizado. El grupo experimental $(n=155)$ consumió harina de maíz enriquecida principalmente con proteína de soja, hierro, vitamina A, ácido fólico, zinc y niacina. El grupo control $(\mathrm{n}=153)$ recibió harina sin enriquecer. Los indicadores evaluados fueron: peso, índice de masa corporal (IMC), circunferencia de cintura y niveles de hemoglobina sanguínea El tiempo de intervención fue a los cuatro y seis meses. El análisis estadístico fue descriptivo, bivariado y con modelos de regresión lineal.

Resultados: Los cambios en los niveles de hemoglobina en el grupo experimental fueron de 13,1 a $13,3 \mathrm{mg} / \mathrm{dL}$. El mejoramiento del nivel de hemoglobina lo manifestó Huejutla, la localidad más marginada (de 12,4 a 12,9 $\mathrm{mg} / \mathrm{dL}$ ). En ambos grupos el incremento de peso fue de $900 \mathrm{~g}$. El aumento del IMC fue igual en ambos grupos: 24,2 a $24,9 / \mathrm{kg} / \mathrm{m}^{2}$. En las mujeres que consumieron la harina enriquecida la circunferencia de cintura no varió $(83,0 \mathrm{~cm})$ pero sí la de la cadera (de 94,3 a $94,9 \mathrm{~cm}$ ) .

Conclusiones: La harina enriquecida es un alimento ideal para mujeres de zonas rurales marginadas que tienen bajo peso, están desnutridas y tienen anemia.

Palabras clave: Población rural. Mujeres. Indígena. Anemia. Indice de masa corporal. Harina, maíz.

Correspondencia

María del Refugio Carrasco Quintero

Instituto Nacional de Ciencias Médicas y Nutrición (INCMN)

Vasco de Quiroga 15, Colonia Sección XVl,

Delegación Tlalpan

Código Postal 14000, México Distrito Federal

carrascoquin@hotmail.com

\section{ABSTRACT \\ Effect of Consumption of Corn Flour Enriched with Soja on Nutrition Status of Indigenous Women of Mexico}

Background: The problem of micronutrient deficiency in the diet, in Mexico affects over 28 million people, mainly rural indigenous women. A solution is food supplementation. The aim of the study was to evaluate the impact of an enriched corn flour soy protein among indigenous women.

Method: The study was conducted with a sample of 308 rural Mexican women, conducted in 2010, longitudinal intervention was double-blind and randomized. The experimental group $(\mathrm{n}=155)$ consumed fortified maize meal (soy protein, iron and vitamin A, folic acid, zinc and niacin). The control group $(n=153)$ received unfortified flour. The evaluated indicators were: weight, body mass index, and waist circumference and blood hemoglobin levels. The intervention time was four months (intermediate) and six months (final). Statistical analysis was descriptive, bivariate and linear regression models.

Results: Changes in hemoglobin levels in the experimental group were 13,1 to $13,3 \mathrm{mg} / \mathrm{dL}$. The improvement of hemoglobine levels was shown by the most marginalized community (from 12,4 to $12,9 \mathrm{mg} / \mathrm{dL}$ ). In both groups, the weight gain was $900 \mathrm{~g}$. Though BMI of the experimental group $(24,2$ to $24,9 / \mathrm{kg} / \mathrm{m} 2)$ with an increase of $(0,7 / \mathrm{kg} / \mathrm{m} 2)$ was equal in both groups, the waist circumference in women who consumed fortified flour was equal at the beginning and end of the study $(83,0 \mathrm{~cm})$ but the hip circumference changed $(94,3$ to $94,9 \mathrm{~cm})$ in women who consumed enriched flour.

Conclusions: enriched flour is an ideal food for marginalized women in rural areas who are underweight, are undernourished and have anemia.

Keyword:Rural people. Women. Indigenous. Anemia. Body mass index. Flour, corn. 


\section{INTRODUCCIÓN}

Uno de los grandes problemas en la dieta es la deficiencia de micronutrientes. Al menos mil millones de la población mundial la sufre ${ }^{1}$. En México esta carencia afecta al $24,5 \%$ de la población, especialmente en zonas rurales y particularmente a mujeres indígenas ${ }^{2}$. En este contexto una consecuencia frecuente es tener anemia, problema común en las zonas marginadas del país. El tipo de dieta que han llevado en las áreas rurales ha cambiado a lo largo del tiempo, sin embargo los cambios no han podido alcanzar un equilibrio en las recomendaciones nutricionales ${ }^{3}$. Actualmente las mujeres de zonas rurales siguen teniendo problemas con su alimentación, por ello es que se crea la necesidad de incrementar alternativas de apoyo alimentario ${ }^{4}$.

El estado nutricio de cualquier población es uno de los principales indicadores para conocer el nivel de salud y bienestar de sus habitantes, por ello su empleo ha sido de gran utilidad ${ }^{5}$. México es un país con grandes contrastes nutricionales, por un lado existe elevada prevalencia de sobrepeso, obesidad y en su contraparte aun predomina la desnutrición y las deficiencias nutricionales específicas (anemia), particularmente en zonas rurales e indígenas ${ }^{6}$.

El estado nutricional nos da la oportunidad de detectar los problemas actuales, como los que está viviendo México ${ }^{7}$. Para evaluarlo se han utilizado diversas mediciones antropométricas, como el peso, la estatura y el perímetro de la cintura y la cadera, siendo estos últimos los que indican la proporción de grasa corporal ${ }^{8}$.

Otro importante indicador del estado de nutrición es la medición de los niveles de hemoglobina, que detectan la anemia, un problema que afecta principalmente durante la infancia y a las mujeres en edad reproductiva ${ }^{9}$. En su caso particular se sabe que a partir de los 15 años la prevalencia es mayor en relación a los hombres, lo cual se debe a la menstruación y a los embarazos ${ }^{10}$. En la Encuesta Nacional de Salud y Nutrición (ENSANUT) se estimó que la prevalencia de anemia en las mujeres no embarazadas era de $11,6 \%$, mientras que para las mujeres embarazadas fue del 17,9\%. También se ha estimado que el porcentaje de anemia de la población de mujeres rurales entre los $12 \mathrm{y}$ 49 años es del $20,5 \%{ }^{7}$.

Se sabe que las zonas rurales es donde mayor atención se requiere en vivienda, salud, educación y alimentación. En México más del 90\% de las localidades siguen siendo rurales (comunidades con una población menor a 2.500 habitantes). De acuerdo al último Censo de Población y Vivienda (INEGI, 2010), el 23,2\% de la población es considerada rural ${ }^{11}$. Sin embargo aún hay entidades federativas que observan un porcentaje de población rural mayor a la media nacional, por ejemplo Hidalgo tiene el 47,8\%,Veracruz el 39,0 \% y el Estado de México el 13,0\%. Ahora bien, a nivel municipal la proporción de población rural es aún mayor que la de su promedio estatal correspondiente: $59,1 \%$ en Huejutla Hidalgo, $42,6 \%$ en Huatusco Veracruz y $39,3 \%$ en Atlacomulco Edo. de México (municipios estudiados) $^{12}$.

Ante la delicada situación alimentaria y nutricional se han implementado diferentes tipos de acciones de gobierno. A partir del período de 1997 al 2010 existieron diversos programas gubernamentales alimentarios, sociales o productivos, encaminados a mejorar la nutrición de la población mexicana. Sin embargo, no ha sido suficiente porque no toda la población fue beneficiaria o no fue elegible en alguno de estos programas.

El enriquecimiento de los alimentos ${ }^{13}$ es una acción que forma parte de algunos programas gubernamentales con enfoques más específicos sobre el estado de nutrición existente ${ }^{14}$. El proyecto que aquí se reporta 
respondió a la necesidad de identificar estrategias que contribuyan a la erradicación de la desnutrición y las deficiencias de nutrimentos en mujeres de zonas rurales.

Se sabe que no hay necesidad de enriquecer los alimentos cuando la dieta consumida es variada y se encuentra en cantidades adecuadas para el ser humano sano y normal. Sin embargo, cuando se tienen dietas carentes en energía y de algunos nutrientes es útil su enriquecimiento ${ }^{15}$.

Existen varios alimentos que han sido enriquecidos, como el maíz, que tiene especial relevancia en México, ya que es la base de la alimentación en la mayoría de la población ${ }^{16}$. En este contexto es evidente que los alimentos básicos tienen un papel importante en los hábitos alimentarios de la población, sobretodo los de zonas rurales que aún es muy extensa. El maíz se ha consumido en México desde épocas prehispánicas $^{11}$ y sigue siendo una de las fuentes principales de energía y otros nutrimentos. La harina de maíz es un alimento fácil de enriquecer al mejorar el aporte nutritivo e incrementando el beneficio de la población que lo consume ${ }^{17}$.

Una opción para ayudar a optimizar la nutrición de la población rural es adicionar nutrimentos (proteína, vitaminas y minerales) a un alimento común (harina de maíz).

El objetivo del presente estudio fue evaluar el impacto de una harina de maíz enriquecida con proteína de soja, hierro, vitamina $\mathrm{A}$, ácido fólico, zinc y niacina en relación al estado de nutrición y niveles de hemoglobina de una población vulnerable de mujeres, tomando en cuenta la etnia, edad, así como la localidad a la que pertenecen. La hipótesis que se planteó es que las mujeres que recibirían la harina enriquecida presentarían mayor incremento de los niveles de hemoglobina y mejorarían su estado de nutrición sin incrementar su peso corporal.

\section{SUJETOS Y MÉTODOS}

Se realizó un estudio longitudinal, aleatorizado y doble ciego, con una muestra de 308 mujeres de las localidades de los municipios de Huejutla de Reyes (Hidalgo), Atlacomulco (Estado de México) y Huatusco (Veracruz). Estas comunidades fueron seleccionadas por estar clasificadas como de alta marginación y por ser rurales (menos de 2.500 habitantes) de acuerdo al Consejo Nacional de Población (CONAPO $2010)^{18}$. Para la participación en el estudio fue necesario que las mujeres residieran en los municipios antes mencionados, tuvieran entre 14 y 64 años. Las mujeres participantes eran tanto indígenas (hablan idioma distinto al español) como no indígenas (hablan solo español).

Previa aprobación del Comité de Ética del Instituto Nacional de Ciencias Médicas y Nutrición se llevó a cabo la realización del proyecto. En cada comunidad se explicó a las mujeres los objetivos y procedimientos del estudio. Se obtuvo el consentimiento informado por escrito de cada una. La intervención consistió en dar a las mujeres del grupo experimental una harina enriquecida $\mathrm{y}$ a las del grupo control harina sin enriquecer. La selección para cada grupo se hizo de forma aleatoria. Ni las mujeres ni los investigadores sabían cuál era la harina enriquecida y cuál sin enriquecer. A cada mujer se le daba mensualmente $20 \mathrm{~kg}$ de harina, 155 recibieron harina enriquecida y 153 sin enriquecer. La harina enriquecida contenía por cada $100 \mathrm{~g}$ : proteína de soja $(1,5 \mathrm{~g})$, hierro (fumarato ferroso $42,4 \mathrm{mg}$ ), vitamina A (120 mcg), ácido fólico (548 mcg), zinc $(33,3 \mathrm{mg})$ y niacina $(6,5 \mathrm{mg})$. Se eligió la soja por ser una leguminosa con alta cantidad de proteína, bajo costo y fácil acceso. El estudio tuvo una duración de diez meses (de enero a octubre de 2010), con tres evaluaciones: al inicio (antes de recibir la harina), intermedia (al cuarto mes) y final. En cada entrega (mensual) se analizaba la harina para verificar las cantidades correctas de 
enriquecimiento. En cada localidad había una nutrióloga y una promotora de salud que hacían visitas domiciliarias para verificar que se estuviera consumiendo la harina, evaluación que se realizó cualitativamente por medio de las preparaciones (tortillas, tlacoyos, atole, tamales, molotitos, pinole, sopas, galletas etc.).

En cada evaluación se midieron el peso, el perímetro de la cintura y de la cadera. La estatura solo se midió al inicio del estudio, clasificándose como normal $\geq-1 \leq 1$ y baja $\geq-2<-1$.E. También se determinó la concentración de hemoglobina (normal 12 $\mathrm{g} / \mathrm{dL}$ y anemia $\leq 11,9 \mathrm{~g} / \mathrm{dL})^{19}$. Las mediciones antropométricas realizadas fueron hechas por las nutriólogas capacitadas y evaluadas según técnicas estandarizadas ${ }^{20}$. A partir del peso y la estatura se estimó el índice de masa corporal $\left(\mathrm{IMC}=\right.$ peso $/ \mathrm{talla}^{2}$ ). No se tomaron pliegues por cuestiones culturales de la comunidad, ya que las mujeres rechazaron el uso del plicómetro.

Se obtuvo una muestra de sangre capilar para medir la concentración de hemoglobina, indicador básico en la práctica por ser el mejor reflejo de las manifestaciones clínicas $^{21}$ y por el tipo de método utilizado, fotómetro portátil (HemoCueMR) el cual resulta exacto e ideal $^{22}$ para trabajar en zonas rurales.

Como variables modificadoras del efecto se consideraron la edad, la talla, la condición de indigenismo y el municipio de residencia. Para el análisis descriptivo se estimaron las medias y los valores máximos y mínimos de cada variable. Para saber si existieron diferencias estadísticamente significativas entre las mujeres del grupo control y del grupo experimental se estimó la t de student para muestras independientes. Dado el carácter longitudinal del estudio se estimaron modelos de regresión lineal para mediciones repetidas, en los cuales las variables dependientes fueron el peso, las circunferencias de cintura y cadera y los niveles de hemoglobina. En los modelos se estimaron las interacciones posibles de la exposición de interés (harina enriquecida) con las variables modificadoras (edad, condición de indigenismo y municipio). Para el análisis bivariado se consideraron las diferencias estadísticamente significativas cuando $\mathrm{p}<0,050$. En los modelos de regresión se estimó que una interacción era estadísticamente significativa cuando $\mathrm{p}<0,100$, criterio sugerido por Méndez ${ }^{23}$.

\section{RESULTADOS}

En la tabla 1 se observa que el porcentaje más alto en edad $(27,6 \%)$ lo tienen las mujeres de 21 a 25 años. El 88,6\% de las mujeres eran indígenas y el $59,1 \%$ presentaron talla baja: $(\leq 149,9 \mathrm{~cm})$.

En la tabla 2 se muestran las medias en los tres momentos de evaluación de acuerdo a la localidad, la etnia, estatura y tipo de harina. La hemoglobina, en general, se incrementó de la segunda a la tercera evaluación de 13,0 mg/dL a 13,2 mg/ dL. Esta misma tendencia se observó en las localidades de Huejutla (de 12,2 a 12,9 g/dL) y Atlacomulco (de 13,8 a 14,0 g/dL). En la comunidad de Huatusco existió una disminución (de 12,6 a 12,5 g/ dL). El aumento del nivel de hemoglobina fue mayor en la tercera medición en la población indígena $(13,0$ versus $13,1 \mathrm{mg} / \mathrm{dL})$ y para las que recibieron la harina enriquecida $(13,1$ versus $13,3 \mathrm{mg} / \mathrm{dL}$ ) y para aquellas que tenían estatura baja. (13,0 versus 13,1 mg/dL). En las mujeres que no recibieron la harina enriquecida existió una reducción de los niveles de hemoglobina (13,1 a 12,9 $\mathrm{mg} / \mathrm{dL}$ ) entre la basal y segunda evaluación, lo mismo paso con las mujeres de estatura normal (13,3 a 13,2 mg/dL).

En el grupo experimental (54,4 a 55,3 $\mathrm{kg})$ y control $(54,5$ a $55,4 \mathrm{~kg})$ existió un incremento de peso, igual que en el IMC el grupo experimental $(24,3$ a $24,7 \mathrm{~kg} / \mathrm{m} 2)$ y del grupo control $(24,6$ a $25,0 \mathrm{~kg} / \mathrm{m} 2)$. El grupo experimental no existieron diferen- 
Tabla 1

\section{Características descriptivas} de las mujeres evaluadas

\begin{tabular}{|c|c|c|}
\hline Localidad & $\mathrm{n}$ & $\%$ \\
\hline Huejutla de Reyes, Hidalgo & 86 & 27,9 \\
\hline Atlacomulco, Estado de México & 127 & 41,2 \\
\hline Huatusco, Veracruz & 95 & 30,8 \\
\hline \multicolumn{3}{|l|}{ Edad } \\
\hline 14-20 años & 45 & 14,6 \\
\hline 21-25 años & 85 & 27,6 \\
\hline 26-30 años & 74 & 24,0 \\
\hline 31- 35 años & 59 & 19,2 \\
\hline 36- 64 años & 45 & 14,6 \\
\hline \multicolumn{3}{|l|}{ Etnia } \\
\hline Indígena & 273 & 88,6 \\
\hline No indígena & 35 & 11,4 \\
\hline \multicolumn{3}{|l|}{ Suplementación de harina } \\
\hline No enriquecida & 153 & 49,7 \\
\hline Enriquecida & 155 & 50,3 \\
\hline \multicolumn{3}{|l|}{ Estatura } \\
\hline Baja & 182 & 59,1 \\
\hline Normal & 126 & 40,9 \\
\hline
\end{tabular}

cias de la medición basal y final $(83,0 \mathrm{~cm})$, pero sí para el grupo control $(83,4$ a 83,5 $\mathrm{cm})$. El aumento en estas variables se verificó en todos los subgrupos formados de acuerdo a la localidad, la etnia, el consumo de harina enriquecida y la estatura. No existieron diferencias estadísticamente significativas entre la población total y ambos grupos en ninguno de los tres momentos evaluados respecto al promedio de circunferencia de cintura $(83,2,83,2$ y $83,3 \mathrm{~cm}$ respectivamente). Las mujeres que no eran indígenas observaron un incremento de la primera a la tercera evaluación de 84,3 a $85,1 \mathrm{~cm}$, es decir, un promedio de la circunferencia de cintura de $0,8 \mathrm{~cm}$. En la localidad de Atlacomulco el incremento también se observó de $0,4 \mathrm{~cm}$.
En la tabla 3 se muestran las medias estimadas de los modelos de regresión para medidas repetidas. Puede apreciarse que en Huejutla y en Atlacomulco existió incremento de los niveles de hemoglobina (de $12,6$ a $13,0 \mathrm{~g} / \mathrm{dL}$ y de 13,9 a $14,1 \mathrm{~g} / \mathrm{dL})$, mientras que en Huatusco se mostró una bajada de $0,4 \mathrm{~g} / \mathrm{dL}$. Las mujeres no indígenas, de talla baja y que consumieron harina enriquecida tuvieron un incremento de 0,1 $\mathrm{g} / \mathrm{dL}$.

En los modelos de regresión se observó que los predictores del cambio en el peso fueron la harina, la localidad, la estatura y la edad (tabla 3). Las mujeres de Atlacomulco pesaban al inicio $58,9 \mathrm{~kg}$, aunque los incrementos de peso fueron más marcados en las de Huejutla (de 51,2 a 53,1 kg) y en Huatusco (de 51,6 a $53,2 \mathrm{~kg}$ ). La ganancia de peso fue mayor $(2,0 \mathrm{~kg})$ en quienes tenían talla normal y eran las más jóvenes (14 a 20 años). En relación al consumo de harina enriquecida el peso fue de $1,5 \mathrm{~kg}$ y con la no enriquecida fue de $1,6 \mathrm{~kg}$, es decir $100 \mathrm{~g}$ de diferencia que no fue estadísticamente significativa.

En relación al IMC (tabla 3) en las mujeres de Atlacomulco tuvo valores más altos al inicio $\left(26,1 \mathrm{~kg} / \mathrm{m}^{2}\right)$ pero el incremento en el tiempo fue mayor en las otras dos localidades $\left(0,9 \mathrm{~kg} / \mathrm{m}^{2}\right.$ en Huejutla, 0,7 en Huatusco y $0,5 \mathrm{~kg} / \mathrm{m}^{2}$ en Atlacomulco $(\mathrm{F}=11,83$ $\mathrm{p}<0,000)$. El incremento del IMC fue mayor en las mujeres más jóvenes (de 14 a 30 años). Se registró el mismo incremento en las mujeres que consumieron la harina enriquecida y $\sin$ enriquecer $\left(0,7 \mathrm{~kg} / \mathrm{m}^{2}\right)$.

Para el perímetro de la cintura, los predictores de los cambios fueron la localidad y la edad (tabla 3). La ganancia fue mayor en Atlacomulco $(0,7 \mathrm{~cm})$, mientras que en las otras dos localidades existió un aumento ( $0,2 \mathrm{~cm}$ en Huejutla y 0,3 en Huatusco). En las mujeres de 14-20 años se registró un mayor aumento $(1,1 \mathrm{~cm})$, aunque esta diferencia no fue estadísticamente significativa. 
Tabla 2

Medias de variables antropométricas en la población total y de acuerdo a localidad, etnia, estatura y tipo de harina

\begin{tabular}{|c|c|c|c|c|c|c|c|c|c|c|c|}
\hline \multirow{3}{*}{ Variables } & \multirow{2}{*}{\multicolumn{2}{|c|}{ Población total }} & \multicolumn{3}{|c|}{ Localidad } & \multicolumn{2}{|c|}{ Indígena } & \multicolumn{2}{|c|}{ Estatura } & \multicolumn{2}{|c|}{ Harina } \\
\hline & & & \multirow{2}{*}{$\frac{\text { HR }}{\text { Media }}$} & \multirow{2}{*}{\begin{tabular}{|c} 
AT \\
Media
\end{tabular}} & \multirow{2}{*}{\begin{tabular}{|c} 
HU \\
Media
\end{tabular}} & \multirow{2}{*}{$\begin{array}{c}\text { Sí } \\
\text { Media }\end{array}$} & \multirow{2}{*}{\begin{tabular}{|c|} 
No \\
Media
\end{tabular}} & \multirow{2}{*}{$\begin{array}{c}\text { Baja } \\
\text { Media }\end{array}$} & \multirow{2}{*}{\begin{tabular}{|l|} 
Normal \\
Media \\
\end{tabular}} & \multirow{2}{*}{\begin{tabular}{|c|}
$\begin{array}{c}\sin \\
\text { enriquecer }\end{array}$ \\
Media \\
\end{tabular}} & \multirow{2}{*}{$\frac{\text { enriquecida }}{\text { Media }}$} \\
\hline & Media & Min/ Máx & & & & & & & & & \\
\hline \multicolumn{12}{|l|}{ Hemoglobina } \\
\hline Medición 1 & 13,1 & $7,7 / 16,0$ & 12,4 & 13,8 & 12,8 & 13,0 & 14,1 & 13,0 & 13,3 & 13,1 & 13,1 \\
\hline Medición 2 & $13,0 \mathrm{~b}$ & $9,4 / 16,0$ & $12,2 b$ & $13,8 b$ & 12,6 & $12,9 b$ & 14,0 & $12,9 b$ & $13,2 b$ & $12,9 \mathrm{~b}$ & $13,1 b$ \\
\hline Medición 3 & $13,2 \mathrm{c}$ & $9,2 / 16,0$ & $12,9 \mathrm{c}$ & $14,0 \mathrm{c}$ & $12,5 \mathrm{c}$ & $13,1 \mathrm{c}$ & 14,2 & $13,1 \mathrm{c}$ & 13,4 & 13,2 & $13,3 \mathrm{c}$ \\
\hline \multicolumn{12}{|l|}{ Peso } \\
\hline Medición 1 & $54,4 \mathrm{a}$ & $33,0 / 99,7$ & $50,8 \mathrm{a}$ & $59,3 \mathrm{a}$ & 51,3 & $54,2 \mathrm{a}$ & 56,3 & $51,4 \mathrm{a}$ & $58,8 \mathrm{a}$ & $54,5 \mathrm{a}$ & $54,4 \mathrm{a}$ \\
\hline Medición 2 & $55,0 \mathrm{~b}$ & $32,9 / 96,9$ & 51,7 & $59,7 \mathrm{~b}$ & $51,6 \mathrm{~b}$ & $54,7 \mathrm{~b}$ & $57,2 b$ & $51,8 \mathrm{~b}$ & $59,6 \mathrm{~b}$ & $55,0 \mathrm{~b}$ & $55,0 \mathrm{~b}$ \\
\hline Medición 3 & $55,3 \mathrm{c}$ & $34,0 / 96,0$ & $52,0 \mathrm{c}$ & $60,0 \mathrm{c}$ & $52,1 \mathrm{c}$ & $55,0 \mathrm{c}$ & $57,7 \mathrm{c}$ & $52,0 \mathrm{c}$ & $60,1 \mathrm{c}$ & $55,4 \mathrm{c}$ & $55,3 \mathrm{c}$ \\
\hline \multicolumn{12}{|l|}{ IMC } \\
\hline Medición 1 & $24,4 \mathrm{a}$ & $14,9 / 43,4$ & $23,2 \mathrm{a}$ & $26,1 \mathrm{a}$ & 23,4 & $24,2 \mathrm{a}$ & 26,0 & $24,3 a$ & $24,6 \mathrm{a}$ & $24,6 \mathrm{a}$ & $24,3 \mathrm{a}$ \\
\hline Medición 2 & $24,7 \mathrm{~b}$ & $14,7 / 42,2$ & 23,6 & $26,2 b$ & $23,5 b$ & $24,4 \mathrm{~b}$ & $26,4 b$ & $24,5 b$ & $25,0 \mathrm{~b}$ & $24,8 b$ & $24,5 b$ \\
\hline Medición 3 & $24,8 \mathrm{c}$ & $14,8 / 41,6$ & $23,8 \mathrm{c}$ & $26,4 \mathrm{c}$ & $23,7 \mathrm{c}$ & $24,6 \mathrm{c}$ & $26,7 \mathrm{c}$ & $24,6 \mathrm{c}$ & $25,2 \mathrm{c}$ & $25,0 \mathrm{c}$ & $24,7 \mathrm{c}$ \\
\hline \multicolumn{12}{|l|}{ Cintura } \\
\hline Medición 1 & 83,2 & $61,0 / 134,0$ & 81,2 & 86,5 & 80,8 & 83,1 & $84,3 \mathrm{a}$ & 82,2 & 84,7 & 83,4 & 83,0 \\
\hline Medición 2 & 83,2 & $58,3 / 132,0$ & 81,0 & 86,7 & 80,5 & 83,0 & 85,1 & 82,1 & 84,8 & 83,5 & 82,9 \\
\hline Medición 3 & 83,3 & $61,0 / 132,0$ & 81,0 & $86,9 \mathrm{c}$ & 80,6 & 83,0 & $85,1 \mathrm{c}$ & 82,1 & 85,0 & 83,5 & 83,0 \\
\hline \multicolumn{12}{|l|}{ Cadera } \\
\hline Medición 1 & $94,3 \mathrm{a}$ & $76,0 / 136,0$ & $91,2^{\mathrm{a}}$ & $98,4^{\mathrm{a}}$ & $91,7 \mathrm{a}$ & $94,0 \mathrm{a}$ & $96,8 \mathrm{a}$ & $92,5 \mathrm{a}$ & $97,0 \mathrm{a}$ & $94,4 a$ & $94,3 \mathrm{a}$ \\
\hline Medición 2 & 94,8 & $75,3 / 133,0$ & 91,7 & 98,8 & $92,2 b$ & $94,5 b$ & 97,6 & 92,8 & 97,7 & 94,9 & 94,8 \\
\hline Medición 3 & $94.9 \mathrm{c}$ & $76.0 / 133.0$ & $91.9 \mathrm{c}$ & $98.8 \mathrm{c}$ & $92.5 \mathrm{c}$ & $94.6 \mathrm{c}$ & $97.4 \mathrm{c}$ & $92.9 \mathrm{c}$ & $97.9 \mathrm{c}$ & $95.0 \mathrm{c}$ & $94.9 \mathrm{c}$ \\
\hline
\end{tabular}

Min./Máx., valores mínimo y máximo; HR, Huejutla de Reyes, Hidalgo; AT, Atlacomulco, Estado de México; HU, Huatusco, Veracruz; IMC, índice de masa corporal. a Diferencias significativas $(\mathrm{p}<0.050)$ entre la primera y la segunda medición. b Diferencias significativas $(\mathrm{p}<0.050)$ entre la segunda y la tercera medición. c Diferencias significativas $(p<0.050)$ entre la primera y la tercera medición.

Para los cambios en la circunferencia de cadera (tabla 3 ), los predictores fueron la localidad, la estatura y la edad. Respecto a la localidad, los incrementos fueron mayores en Huejutla $(1,0 \mathrm{~cm})$, Huatusco $(1,2 \mathrm{~cm})$ y en Atlacomulco $(0,5 \mathrm{~cm})$. Respecto a la estatura, las que estaban normal tuvieron un incremento de $1,3 \mathrm{~cm}$ y para las de talla baja de $0,6 \mathrm{~cm}$. Las mujeres más jóvenes tuvieron mayor incremento de la circunferencia de cadera $1,6 \mathrm{~cm}$ versus $0,4 \mathrm{~cm}$ en comparación de las de mayor edad (36-64años). 
Tabla 3

Evolución de las variables dependientes en las diferentes intervenciones

\begin{tabular}{|c|c|c|c|c|c|c|c|c|c|}
\hline \multirow[b]{2}{*}{ Variable } & \multicolumn{3}{|c|}{ Localidad } & \multicolumn{2}{|c|}{ Indígena } & \multicolumn{2}{|c|}{ Estatura } & \multicolumn{2}{|c|}{ Harina } \\
\hline & HR & AT & $\mathrm{HU}$ & Sí & No & Baja & Normal & $\begin{array}{c}\text { No } \\
\text { enriquecida }\end{array}$ & Enriquecida \\
\hline \multicolumn{10}{|l|}{ Hemoglobina } \\
\hline Intervención basal & 12,6 & 13,9 & 13,0 & 13,4 & 13,0 & 13,1 & 13,2 & 13,2 & 13,2 \\
\hline Intervención media & 12,3 & 13,9 & 12,7 & 13,1 & 12,9 & 12,9 & 130 & 12,9 & 13,0 \\
\hline Intervención final & 13,0 & 14,1 & 12,6 & 13,3 & 13,1 & 13,1 & 13,2 & 13,1 & 13,3 \\
\hline \multicolumn{10}{|l|}{ Peso } \\
\hline Intervención basal & 51,2 & 58,9 & 51,6 & 52,9 & 54,9 & 50,9 & 56,9 & 54,1 & 53,7 \\
\hline Intervención media & 52,6 & 59,5 & 52,5 & 54,3 & 55,4 & 51,6 & 58,1 & 55,0 & 54,7 \\
\hline Intervención final & 53,1 & 60,0 & 53,2 & 55,2 & 55,7 & 51,6 & 58,9 & 55,7 & 55,2 \\
\hline \multicolumn{10}{|l|}{ IMC } \\
\hline Intervención basal & 23,3 & 26,1 & 23,6 & 24,3 & 24,3 & 24,4 & 24,3 & 24,4 & 24,2 \\
\hline Intervención media & 24,0 & 26,4 & 24,0 & 25,0 & 24,6 & 24,7 & 24,8 & 24,9 & 247 \\
\hline Intervención final & 24,2 & 26,6 & 24,3 & 25,4 & 25,7 & 24,9 & 25,2 & 25,1 & 24,9 \\
\hline \multicolumn{10}{|l|}{ Cintura } \\
\hline Intervención basal & 80,3 & 85,8 & 80,0 & 81,9 & 82,3 & 81,4 & 82,7 & 82,3 & 81,9 \\
\hline Intervención media & 80,7 & 86,4 & 80,4 & 82,1 & 82,8 & 81,7 & 83,3 & 82,8 & 82,1 \\
\hline Intervención final & 80,5 & 86,5 & 80,3 & 82,1 & 82,7 & 81,6 & 83,2 & 82,7 & 82,1 \\
\hline \multicolumn{10}{|l|}{ Cadera } \\
\hline Intervención basal & 91,3 & 98,0 & 91,7 & 81,9 & 82,3 & 92,1 & 95,2 & 82,3 & 81,9 \\
\hline Intervención media & 92,3 & 98,5 & 92,8 & 82,1 & 82,8 & 92,7 & 96,4 & 82,8 & 82,1 \\
\hline Intervención final & 92,3 & 98,5 & 92,9 & 82,1 & 82,7 & 92,7 & 96,5 & 82,7 & 82,1 \\
\hline
\end{tabular}

HR, Huejutla de Reyes, Hidalgo; AT, Atlacomulco, Estado de México; HU, Huatusco, Veracruz; IMC, índice de masa corporal.

\section{DISCUSIÓN}

La harina de maíz enriquecida demostró ser un alimento eficaz para mejorar el estado de nutrición general de la población objeto de estudio, es decir la población más vulnerable y afectada por una problemática alimentaria generacional, que se manifiesta, entre otras cosas, por una talla baja. En este contexto más de la mitad de las mujeres del estudio tenían una talla inferior a los estándares corres- pondientes. Situación avalada con el estudio de Mardones y col. ${ }^{24}$ en el que se comprueba que la estatura baja está estrechamente relacionada con factores ambientales, más que con los genéticos. Efecto que también tiene que ver más con carencias durante la infancia según lo reportado, por las investigaciones de Torres y col. ${ }^{25}$. Por lo tanto, los hallazgos del trabajo de campo son de gran utilidad porque las comunidades estudiadas son representativas de las zonas indígenas y 
del México rural, que son a su vez las regiones más afectadas del país.

Otro importante indicador del estudio fue la hemoglobina, que registró un incremento en sus niveles en las mujeres que consumieron la harina enriquecida. Este hallazgo también lo reporta Haro y col. ${ }^{13}$ en su trabajo, en el que dice que el enriquecimiento de los alimentos con hierro es una buena estrategia a largo plazo para reducir la anemia causada por su deficiencia, más común en las mujeres de zonas rurales. Aunque los incrementos de hemoglobina no fueron los óptimos, existieron diferencias cuantitativas pero no estadístícamente significativas con el grupo control. Sin embargo creemos que si la cantidad de nutrimentos utilizados para el enriquecimiento (mínima) y el tiempo durante el que se consumió la harina fueron importantes en el grupo experimental, el impacto sería mayor si el enriquecimiento hubiera sido más alto. De acuerdo a lo reportado por estudios previos de Chávez y David $^{16,26}$ la cantidad de hierro a adicionar y el tiempo son fundamentales para garantizar un mejor beneficio en el consumo del alimento enriquecido. No obstante, parte del objetivo del trabajo era saber si existían diferencias en los niveles de hemoglobina empleando un mínimo de enriquecimiento, lo cual se comprobó.

El peso es otro de los indicadores más sensibles que manifiesta de forma inmediata cualquier cambio que se dé a corto o largo plazo. En el estudio el incremento de peso que se observa en las localidades de Huejutla y Huatusco se debe en gran medida a que en estos lugares es donde mayor carencia alimentaria existe y que al tener acceso a un alimento extra (harina) incrementó su aporte energético y por ende su peso, independientemente de si estaba enriquecida o no. $\mathrm{Al}$ respecto Peña y $\mathrm{col}^{27}$ reportan que los habitantes más pobres, como los del estudio, son los que tienen menos información sobre la alimentación y las repercusiones que se tienen al consumir alimentos alta- mente energéticos (bebidas y frituras) en su cuerpo (sobrepeso u obesidad) y, por ende, en su salud.

Referente a la variación que se dio en el IMC, en las mujeres de Atlacomulco se cree que existe cierta influencia con los hábitos alimentarios que están siguiendo, dado que ya no corresponde a los tradicionales si no que se han modificado por ser el lugar que se encuentra más cercano al Distrito Federal. Esta situación, también la describe Nájera $^{28}$ en su trabajo, donde dice que la alimentación ha tenido transformaciones que incluyen procesos de cambio donde sustituyen alimentos nutritivos por otros que afectan sus prácticas, costumbres y por ende su estado de nutrición.

Los alimentos enriquecidos, como lo reporta Cohen y Franco ${ }^{29}$, son una excelente opción para zonas rurales y poblaciones con deficiencias de nutrimentos. Sin embargo cabe hacer énfasis en que una limitación del estudio está en relación al uso de la harina, que a pesar de ser un alimento adecuado no se puede consumir a libre demanda, particularmente en las personas que tengan sobrepeso u obesidad porque se podría incrementar más el peso, lo cual no sería positivo.

Se concluye en la investigación que la harina enriquecida utilizada fue una buena alternativa al ser un alimento básico para las localidades rurales. En el grupo experimental se tuvo mayor rendimiento en términos de hemoglobina, aunque estos no hayan sido altamente significativos. Respecto a los otros indicadores (peso, IMC, cintura y cadera) tuvo el mismo impacto que en el grupo control, la ventaja del grupo experimental es que la harina enriquecida no incrementa más estas variables y por lo tanto tiene el mismo comportamiento a nivel antropométrico que la harina no enriquecida, con la ventaja de que la harina enriquecida contiene mayor cantidad de nutrimentos. Otro beneficio es que se trata de un pro- 
ducto semiprocesado, de fácil adquisición, preparación y por tanto ideal para poblaciones marginadas, coadyuvando a mejorar el estado nutricional.

Se recomienda que la harina enriquecida sea consumida principalmente por mujeres de bajo peso, desnutridas y anémicas. Para aquellas mujeres que tengan un peso adecuado lo ideal es que la harina sea consumida con mesura y no a libre demanda.

\section{BIBLIOGRAFÍA}

1. Organización de las Naciones Unidas para la Agricultura y Alimentación. El estado de la seguridad alimentaria en el mundo. Roma: FAO; 2012.

2. Consejo Nacional para la Evaluación de la Pobreza (Mx). Informe de Evaluación de la Política Social en México: CONEVAL; 2011.

3. Ortiz Gómez A, Vázquez García V, Montes Estrada M. La alimentación en México: enfoques y visión a futuro. Rev. Invest Cient. 2005; 13 (25): 7 - 34

4. Mejía Rodríguez F, Camacho Cisneros M; García Guerra A, Monterrubio Flores E, Shamah Levy T, Villalpando Hernández S: Factores asociados al uso de suplementos alimenticios en mujeres mexicanas de 12 a 49 años de edad. Rev. Arch Latinoam Nutr. 2008; 58 : 164173.

5. Oswald Spring U. Políticas Alimentarías. En: Derechos Sociales y Desarrollo Incluyente. México: Juan Pablos Editor. Mexico DF:Consejo Nacional de Universitarios por una Estrategia de Desarrollo México; 2012; p. $168-192$

6. Barquera Fernandez S, Rivera Dommarco J, Gasca García A. Políticas y Programas de Alimentación y Nutrición. Rev Salud Pública México. 2001; 43: 464477.

7. Instituto Nacional de Salud Pública (Mx). Encuesta Nacional de Salud y Nutrición. Mexico DF: INSP; 2012.

8. Riobo P, Fernández Bobadilla B, Kozarcewski M y Fernández Moya J. Obesidad en la mujer. Rev Nutr Hosp. 2003; 18 (5): 233-237.

9. Casanueva E, Regil LM, Flores Campuzano. Anemia por deficiencia de hierro en mujeres mexicanas en edad reproductiva. Historia de un problema no resuelto. Rev Salud Pública México 2006; 48: 166-175.
10. Méndez Estrada R, Pacheco B, Noriega Verdugo H Quihui L, Morales G, Valencia J Mauro E. Prevalecía de deficiencia de hierro y de anemia por deficiencia de hierro en adolescentes embarazadas del noroeste de México, 2007- 2008. Rev. Ven ALAN Caracas. 2009: 59 (2): 147-151.

11. Roldán JA, Carrasco MR, Guarneros N. El hambre, parte de la cultura en los hogares marginales de México. $1^{\mathrm{a}}$ ed. Saarbrücken: Editorial Académica Española,; 2012 .

12. Instituto Nacional de Estadística y Geografía, Censo Nacional de Población y Vivienda 2010, (citado 12 de octubre 2011). Disponible en: http://www3.inegi.org.mx/sistemas/TabuladosBasi$\cos /$ Default.aspx? $=27302 \&$ s $=$ est

13. Haro Vicente J, Martínez García, Periago María J, Ros G. Prevención de la deficiencia en hierro mediante el enriquecimiento de los alimentos. Rev Esp An Vet. 2005; 21: 7- 21 .

14. Ávila CA, Flores SJ, Rangel FG. La política Alimentaría en México. $1^{\mathrm{a}}$ ed. México: CEDRSSA; 2011.

15. Scrimshaw Nevins S. La fortificación de alimentos: una estrategia nutricional indispensable. Rev Esp An Vet. . 2005; 18 (1): 64-68.

16. Chávez Villasana A, Muñoz de Chávez M. El maíz y la tortilla en México; sus posibilidades de fortificación. En: La tortilla de Alto valor Nutritivo. Mexico DF: Mc. Graw Hill; 2003. 13 -39.

17. Carrasco Quintero MR, Ortiz Hernández L, Chávez Villasana A, Roldán Amaro J, Guarneros Soto N, Aguirre Arenas J, et al. Impacto en el Consumo de harina de maíz con un bajo nivel de enriquecimiento en niños rurales. Nutr Hosp España. 2011; 26 (5): 1097-1104.

18. Consejo Nacional de Población (Mx). Clasificación de municipios y comunidades de acuerdo a su grado de marginación; México: CONAPO; 2010.

19. Organización Panamericana para la Salud. La anemia entre adolescentes y mujeres adultas en América Latina y el Caribe: Un motivo de preocupación. Washington (DC): OPS;2010.

20. Saucedo AG, Villalpando A, Aguilar C, Chávez A. Manual de Antropometría. $1^{\mathrm{a}}$ ed. México DF: INCNN;2004.

21. Naucapoma EM, Rojas GM: Estudio de los índices eritrocitarios del adulto mayor [tesis profesional]. Lima: Univ Nacional Mayor de San Marcos; 2005. 
22. Neufeld Lynnette, Garcia Guerra A, Sánchez Francia D, Newton Sánchez O, Ramirez Villalobos M, Rivera Dommarco J. Hemoglobin measured by Hemocue and reference method in venous and capillary blood: a validations study. Rev Mex. Salud Pública. 2002; 44: $219-227$

23. Méndez RI. Modelos estadísticos lineales en la investigación comparativa. $1^{\mathrm{a}}$ ed. México DF: Instituto de investigación en matemáticas aplicadas y sistemas; 1995.

24. Mardones S, Mallea A, Villaroel del P, Urrutia S, Rose H, Durán et al. Influencia socioeconómica ética sobre la talla de la mujer Chilena. Rev Chi Nutr. 2004; 31 (3): 296-304.

25. Torres J, Villoro R, Ramírez T, Zurita B, Hernández $\mathrm{P}$, et al. La salud de la población indígena en México. [Citado 14 de marzo de 2013].Disponible en: www.funsalud.org.mx/CASEsalud/caleidoscopio/02\%20PoblacionIndigena.pdf

26. David L. Jorge. Fortificación de harina de trigo en América Latina y región del caribe. Rev Chil Nutr. 2004; 31 (3): 336-347.

27. Peña M, Bacallao J. La obesidad en la pobreza: un problema emergente en las Américas. En: Obesidad en la pobreza, un nuevo reto para la salud pública. Washington: OPS; 2000. 3-11

28. Nájera Castellanos AJ, Álvarez Gordillo G. Del Posol a la Coca Cola: cambios en las prácticas alimentarias en dos comunidades tojolabales de México. Rev Mex Liminar. 2010; 8: 173-190.

29. Cohen E, Franco R. Seguimiento y evaluación de impacto de los programas de protección social basados en alimentos en América Latina y el Caribe. [Citado 14 de mayo de 2013]. Disponible en:ttp://www.enap.gov.br/downloads/ec43ea4fSeguimento_evaluacion_de_impacto_de_los_programas.pdf 